\title{
Development study of new topical formulations with erythromycin
}

\author{
Lacramioara OCHIUZ¹, Andreea CRETEANU¹, Manuela HORTOLOMEI², \\ Catalina Anisoara PEPTU ${ }^{3}$, Iulian STOLERIU4, Marcel POPA ${ }^{5}$, Cristian PEPTU6 \\ ${ }^{1}$ Faculty of Pharmacy, "Grigore T. Popa" University of Medicine and Pharmacy, lasi, Romania \\ ${ }^{2}$ Ester Farm LTD, Iasi Romania \\ ${ }^{3}$ Faculty of Chemical Engineering and Protection of the Environment, "Gheorghe Asachi" Technical University, \\ lasi, Romania \\ ${ }^{4}$ Faculty of Mathematics, "Alexandru loan Cuza" University, lasi, Romania \\ ${ }^{5}$ The Academy of Romanian Scientists, Bucharest, Romania \\ "Petru Poni" Institute of Macromolecular Chemistry, Iasi, Romania
}

\begin{abstract}
Erythromycin is a macrolide antibiotic prescribed for the topical treatment of acne. Pharmacologically, erythromycin has the disadvantage of being poorly soluble in water. This leads to formulation challenges in semisolid dosage forms. In recent years, many published studies have shown the ability of cyclodextrins to form complexes with drugs. These new complexes are characterized by much improved solubility and permeation compared to the "parent" molecule. The aim of this study was to synthesize an inclusion complex of erythromycin and lactide- $\beta$-cyclodextrin for the formulation of semisolid bases and the development of innovative topical preparations with erythromycin. The erythromycin-lactide- $\beta$ cyclodextrin complex was characterized by scanning electron microscopy and Fourier-transform infrared spectroscopy. Semisolid formulations were pharmacologically evaluated by in vitro dissolution test and kinetic analysis of drug release by fitting to representative mathematical models. The results obtained showed a prolonged release of erythromycin from erythromycin-lactide- $\beta$-cyclodextrin formulations and a higher permeability coefficient of these formulations compared to the erythromycin-based release.
\end{abstract}

Keywords: erythromycin, cyclodextrins, semisolid dosage forms

\section{INTRODUCTION}

Local antibiotherapy is one of the most effective acne therapies. Erythromycin (ER) is a macrolide antibiotic administered topically for over 30 years in the form of gels, ointments or hydroalcoholic solutions in acne therapy. The use of ER as a base for topical dosage forms raises some technological challenges due to the physicochemical properties of this substance [1-3]. The main disadvantage of ER is its poor water solubility $(2 \mathrm{mg} /$ $\mathrm{ml}$ ) that limits both formulation using hydrophilic bases and skin permeability [4-5]. Cyclodextrins (CDs) are biocompatible cyclic oligomers of glucose, with a hydrophobic core and a hydrophilic exterior. CDs are used to improve 
the bioavailability of drugs by increasing their solubility and/or their dissolution rate after including the poorly water-soluble substances (such as ER) in the hydrophobic cavity of CDs. Adding CDs leads to improved solubility and stability of the drug substance, increased permeability of low aqueous solubility, decreased toxicity and even to reduced active dose as a result of increased bioavailability. CDs increase skin tolerability by reducing the irritant effect of certain substances [6]. We have included ER to lactide modified $\beta$-cyclodextrin, in order to improve the therapeutic effect of topically administered ER. The aims of the present study were: to synthesize and describe a new complex with prolonged release of ER with lactide modified $\beta$-cyclodextrin (CD-LA_E); to investigate the CD-LA E complex by scanning electron microscopy (SEM) and Fourier transform infrared spectroscopy (FTIR); to analyze the effect of the semisolid base on the in vitro release characteristics of ER in the CD-LA_E complex by assessing the permeability coefficient and the release kinetics by fitting on mathematical models.

\section{MATERIALS AND METHODS}

b-cyclodextrin (Sigma Aldrich, Germany), erythromycin, purity 99.85\% (Zhejlang Sanmen Hengkang Pharmaceutical Co. Ltd. China) lactic acid, alcohol, methanol pharmaceutical purity - were purchased from MedChim Company, Bucharest. Hydroxypropylcellulose (HPC) 150 - $4000 \mathrm{cP}$ was received as a sample from Nisso Chemical Europe $\mathrm{GmbH}$, Germany. Carbopol 940 (C 940), propylene glycol, triethanolamine, cetyl alcohol, petroleum jelly, glycerol monostearate and isopropylmyristate - pharmaceutical purity - were purchased from MedChim Company, Bucharest. Soya lecithin, Lutrol F 127 (127 L) and Lutrol F 68 ( $L$ 68) were donated by BASF Germany. The preparation of gels used pure water and double distilled water (Millipore conductivity $-0.01 \mathrm{Ms} / \mathrm{cm}$ ). All reagents used met the quality requirements of RP $X$. In vitro dissolution tests were conducted on Nylon synthetic membrane with a $50 \mathrm{~mm}$ pore diameter and pores of $\varnothing=45 \mu \mathrm{m}$ (Millipore, Merck Germany).

\section{Preparation of the erythromycin 6-cyclodextrin-lactide complex - (CD-LA_E)}

The polarity of the $\beta-C D$ molecule has been adjusted by preparing lactide modified cyclodextrin (CD-LA). In order to obtain CD-LA, $\beta-C D$ was functionalized with an average of 3 units of lactide. The $\beta-C D$ derivative modified with oligoester lactide (LA) was prepared by LA ring opening exclusively with $\beta-C D$ by Shen method [7]. The complex erythromycin - lactide modified $\beta$-cyclodextrin (CD-LA_E) was prepared following the method described by Song [6]. The work technique consists in preparing a CD-LA aqueous solution, concentration $0.125 \mathrm{~g} / \mathrm{ml}$, and an ER alcoholic solution of $0.25 \mathrm{~g} / \mathrm{ml}$. The two solutions were mixed in a molar ratio of 1:1. The ER solution was added dropwise under continuous stirring for $30 \mathrm{~min}$. The complex obtained was washed three times with alcohol to remove uncomplexed ER. In the last stage, the complex was lyophilized at $-30^{\circ} \mathrm{C}$ after being frozen for 12 hours at $20^{\circ} \mathrm{C}$, in order to remove the solvent completely.

\section{Physical-chemical characterization of the CD-LA_E complex}

Scanning electron microscopy (SEM) was performed using a scanning electron microscope Shimadzu SSX 550 by visualizing a constant sample quantity. Mass spectrometry with electrospray ionization (ESI-MS) is known as a simple and quick method applied to the characterization of $\mathrm{CD}$ derivatives. This method was applied for the characterization of the $\mathrm{CD}$ derivatives obtained by ring-opening and oligomerization with $\beta$-butyrolactone and [8-9], D, L - lactide [7] and other cyclic esters [10]. In our studies we used an AGILENT 6520 QTOF spectrometer equipped with electrospray ionization. The analysis of ESI-MS was performed under the following conditions: $\mathrm{VCAP}=4000 \mathrm{~V}$, fragmentor voltage $=175 \mathrm{~V}$, temperature of drying gas $=325^{\circ} \mathrm{C}$, gas flow $=5 \mathrm{~L} / \mathrm{min}$, nebulization pressure $=$ $35 \mathrm{psig}$, injection flow $=0.5 \mathrm{ml} / \mathrm{min}$. Fourier transform infrared absorption spectroscopy (FT-IR) - carried out with a Vertex 70 FT-IR spectrometer (Bruker, Germany), using the encapsulation technique in $\mathrm{KBr}$. The $\mathrm{KBr}$ pellets were prepared by mixing $3 \mathrm{mg}$ of sample with $500 \mathrm{mg}$ of $\mathrm{KBr}$ of appropriate purity. 128 records were made for each sample, $2 \mathrm{~cm}^{-1}$ resolution in absorbance and transmittance. The spectra obtained were compared with those presented in the literature [11].

\section{Preparation of dermal preparations with CD-LA_E}

CD-LA_E was dispersed in the base, so that the gels have a final concentration of $2 \%$ free erythromycin. We used 6 different bases abbreviated in Table 1. 
TABLE 1. Abbreviation of gel formulations with CD-LA_E

\begin{tabular}{|c|c|}
\hline Gel Formulati & Abbreviati \\
\hline HPC gel, 3,5\% & B1-CE 2\% \\
\hline C 940 gel, 2,5\% & B2-CE 2\% \\
\hline L/H emulsion & B3-CE 2\% \\
\hline $\begin{array}{c}\text { L 127 gel, 15\% } \\
\text { and L 68 gel, 3\% }\end{array}$ & B4-CE 2\% \\
\hline L 127 gel, 13\% & B5-CE 2\% \\
\hline Organogel & B6-CE 2\% \\
\hline
\end{tabular}

\section{Evaluation of in vitro release of erythromycin}

The in vitro dissolution test was performed on an Enhancer cell with a diameter of $2.5 \mathrm{~cm}$, employing an SR 8 Plus Series device (AB \& L Jasco), according to the following protocol: dissolution medium: phosphate buffer $\mathrm{pH} 7.4,100 \mathrm{ml}$; mass of sample: each formulation studied used $0.5 \mathrm{~g}$ Nylon synthetic membrane with a pore diameter $\varnothing$ $=45 \mu \mathrm{m}$ (Millipore, Merck Germany); temperature: $37^{\circ} \mathrm{C} \pm 0.2^{\circ} \mathrm{C}$; harvest interval: the test was carried out over a 12-hour interval; every $60 \mathrm{~min}$, we harvested a sample volume of $1 \mathrm{ml}$, which was replaced with fresh medium; rate: $100 \mathrm{rpm}$.

The synthetic membrane was placed in the dissolution medium for 24 hours, prior to performing the in vitro test, so as to obtain optimal hydration and expansion of the pores. The harvested samples were filtered through a $0.45 \mu \mathrm{m}$ filter, and then chromatographed by High Pressure Liquid Chromatography method [12].

\section{Determination of the permeability coefficient of erythromycin}

The permeability coefficient was calculated by the following equation [13]:

$$
\mathrm{Kp}=\mathrm{J} / \mathrm{C} \times \mathrm{A}
$$

in which:

$K p$ - permeability coefficient $(\mathrm{cm} / \mathrm{h})$;
$J$ - rate of permeation of drug substance or flow in stationary phase $(\mu \mathrm{g} / \mathrm{h})$;

$C$ - concentration in the donor compartment $(\mu \mathrm{g} / \mathrm{ml})$

$A$ - contact surface area $\left(\mathrm{cm}^{2}\right)$

\section{Evaluation of release kinetics of erythromycin}

Experimental data obtained from studies of ER release through biological or synthetic membrane were analyzed by fitting on four mathematical models (zero-order model, first-order model, Higuchi model and Korsmeyer-Peppas model), according to the equations presented by Kierstan et al. and Lucero et al. [14, 15].

Data fitting was performed by linear and nonlinear regression using Matlab 7.1. Data were presented as a mean \pm the standard deviation and were considered statistically significant at $p<0.05$.

\section{RESULTS AND DISCUSSIONS}

The amorphous morphology of the powders of CD-LA and CD-LA_E complex was observed by SEM (Fig. 1.a and Fig. 1.c, respectively.). This morphology is consistent with the data reported in the literature [16]. Pure free ER shows a semicrystalline structure, as shown in Figure 1.b. Thus, the mixing process implemented in the CD preparation step facilitates the interaction of $C D$ with $E R$, with the alteration of the original crystalline structures of these compounds and the formation of an amorphous structure during criodessication, as shown in Fig. 1.c.

$\beta-C D$ was functionalized with a variable number of lactide units from $1(\mathrm{~m} / \mathrm{z}=1296)$ to $6(\mathrm{~m} / \mathrm{z}=$ 2016), with an average of 3 units, as shown in Fig. 2. For example, the peak at $\mathrm{m} / \mathrm{z}=1728$ corresponds to a CD molecule carrying 4 lactide units, according to the following stoichiometric calculation: $1728=$ $1134(\mathrm{CD})+4 \times 144(\mathrm{LA})+18\left(\mathrm{NH}_{4}^{+}\right)$. In the structure of the CD-LA_E complex, the molar ratio between CDLA and ER is 2.12 to 1 .

ER characteristic spectrum shown in Fig. 3.a presents the following specific absorption bands: 3522 $\mathrm{cm}^{-1}$ corresponding to the $-\mathrm{OH}$ groups, $2974 \mathrm{~cm}^{-1}-\mathrm{CH}_{2}, 1715 \mathrm{~cm}^{-1}$ $-\mathrm{C}=\mathrm{O}, 1639 \mathrm{~cm}^{-1}-\mathrm{OH}$ groups, 1464 $\mathrm{cm}^{-1}-\mathrm{N}\left(\mathrm{CH}_{3}\right)_{2}, 1379 \mathrm{~cm}^{-1}-\mathrm{OH}$, $1271 \mathrm{~cm}^{-1}-\mathrm{OH}, 1194 \mathrm{~cm}^{-1}-\mathrm{C}-\mathrm{O}-\mathrm{C}$, $1096 \mathrm{~cm}^{-1}-\mathrm{C}-\mathrm{O} / \mathrm{C}-\mathrm{C}, 1009 \mathrm{~cm}^{-1}-$ $\mathrm{C}-\mathrm{N}, 903 \mathrm{~cm}^{-1}-\mathrm{N}-\mathrm{CH}_{3}$.

The CD-LA spectrum, shown in Fig. 3.b manifests the following specific absorption bands: $3400 \mathrm{~cm}^{-1}-\mathrm{OH}$ groups, $2932 \mathrm{~cm}^{-1}-\mathrm{CH}_{2}, 1747 \mathrm{~cm}^{-1}$ $-\mathrm{C}=\mathrm{O}, 1639 \mathrm{~cm}^{-1}-\mathrm{OH}$ groups, 1456 and $1402 \mathrm{~cm}^{-1}-\mathrm{CH}_{3}$ groups from lactide, $1155 \mathrm{~cm}^{-1}$ - C-O-C, 1080 $\mathrm{cm}^{-1}-\mathrm{OH}$ groups, $1030 \mathrm{~cm}^{-1}-\mathrm{C}-$ $\mathrm{O} / \mathrm{C}-\mathrm{C}$.

The spectrum obtained for the $C D$ - LA_E complex shown in Fig. 3.c allows the observation of distinct absorption bands resulting from the contribution of the mixture components, erythromycin and CD-LA, together with modified peaks due to inclusion of $E R$ in CD-LA. Thus, the bands corresponding to the carbonyl group of ER at $1715 \mathrm{~cm}^{-1}$ were displaced as a result of the inclusion process at 1657 $\mathrm{cm}^{-1}$, the bands observed $1740 \mathrm{~cm}^{-1}$ corresponding to the carbonyl groups of the CD-LA. At the same time, the bands corresponding to the $-\mathrm{OH}$ groups in the ER structure at $1639 \mathrm{~cm}^{-1}$ were displaced by complexation to $1611 \mathrm{~cm}^{-1}$. The results of the in vitro dissolution test showed that ER solubility was greatly enhanced by complexation, the amount of ER released from the gels with CD-LA_E being in the range of 76.23 to $89.01 \%$. (Fig. 4). 


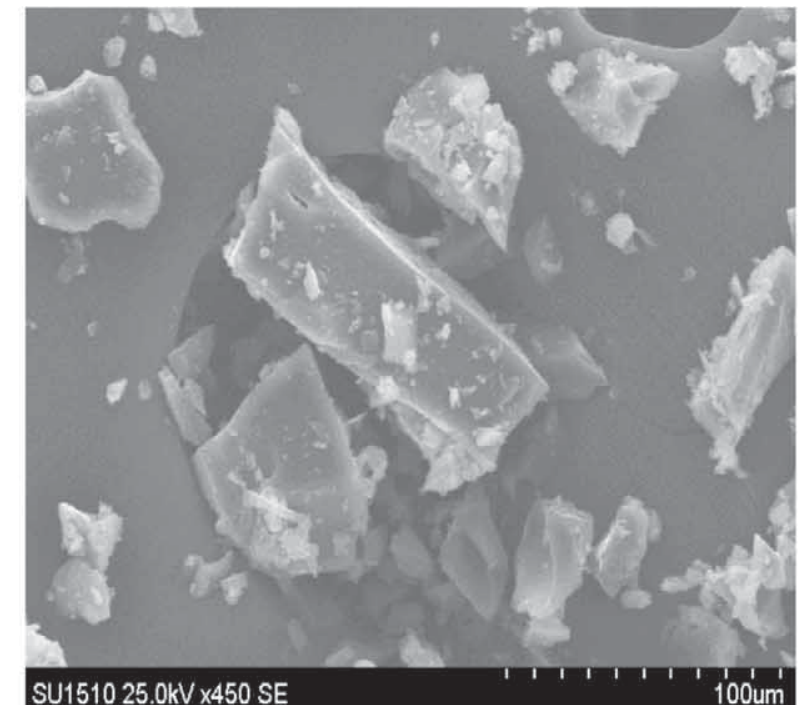

SU1510 25.0kV x450 SE

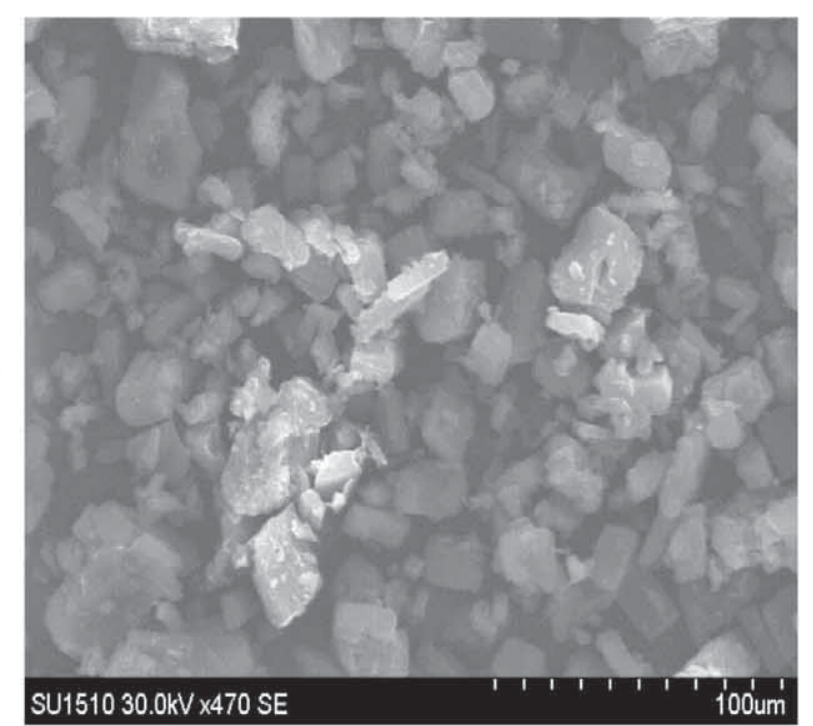

C

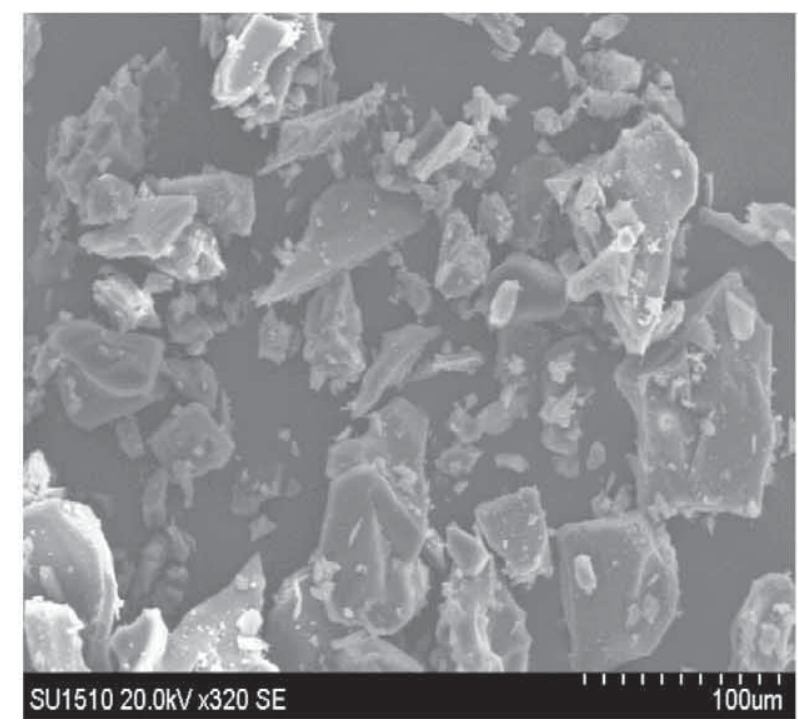

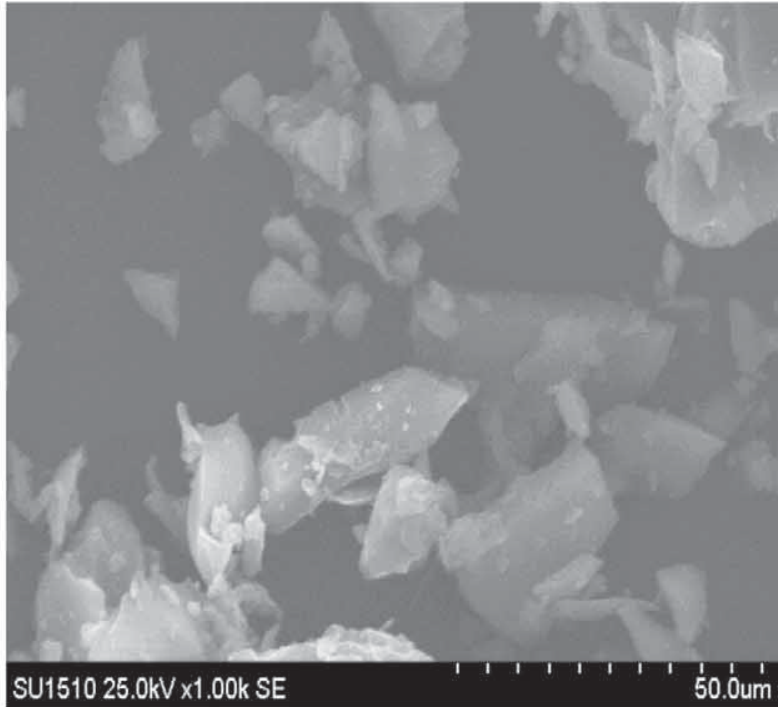
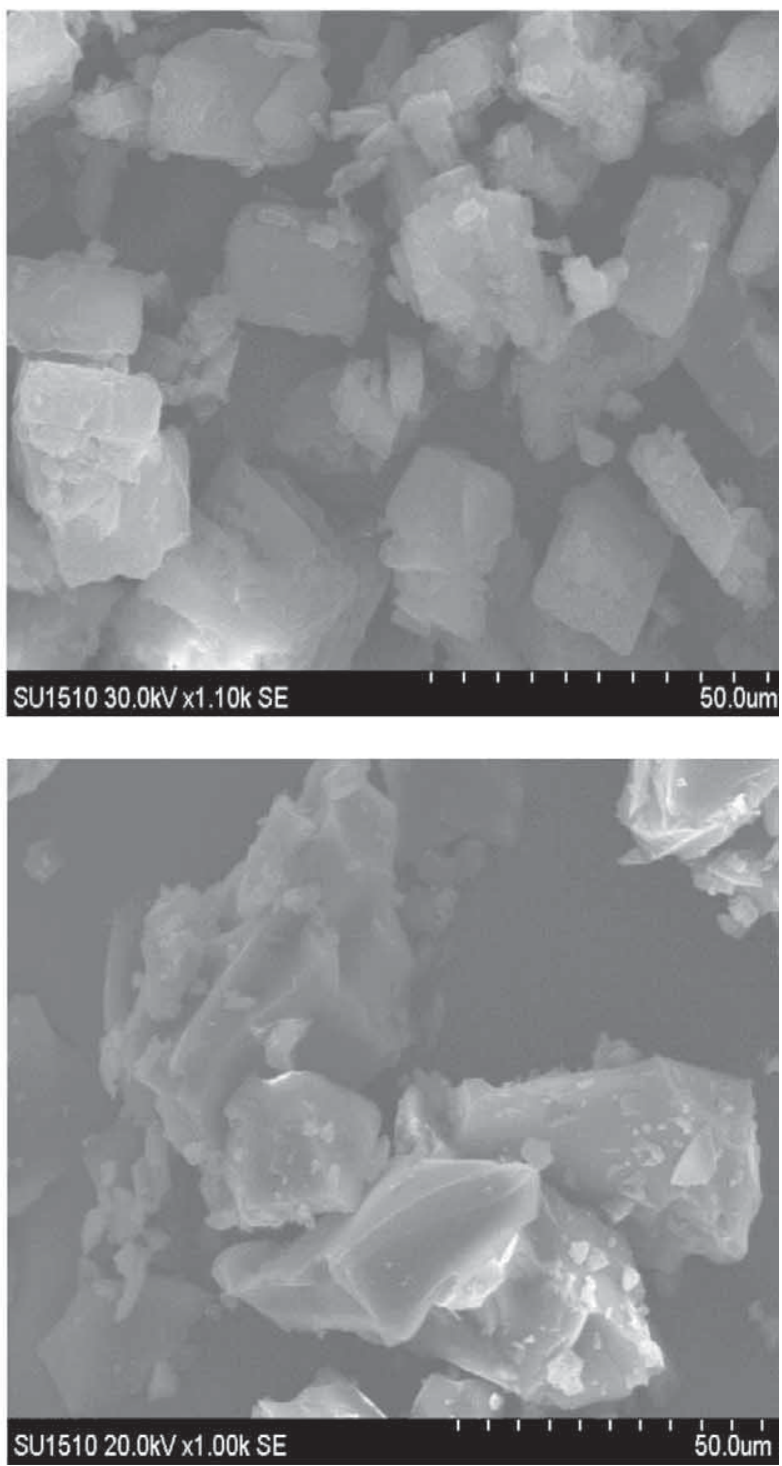

FIGURE 1. SEM image of CD-LA (a), ER (b) and CD-LA_E (c) 


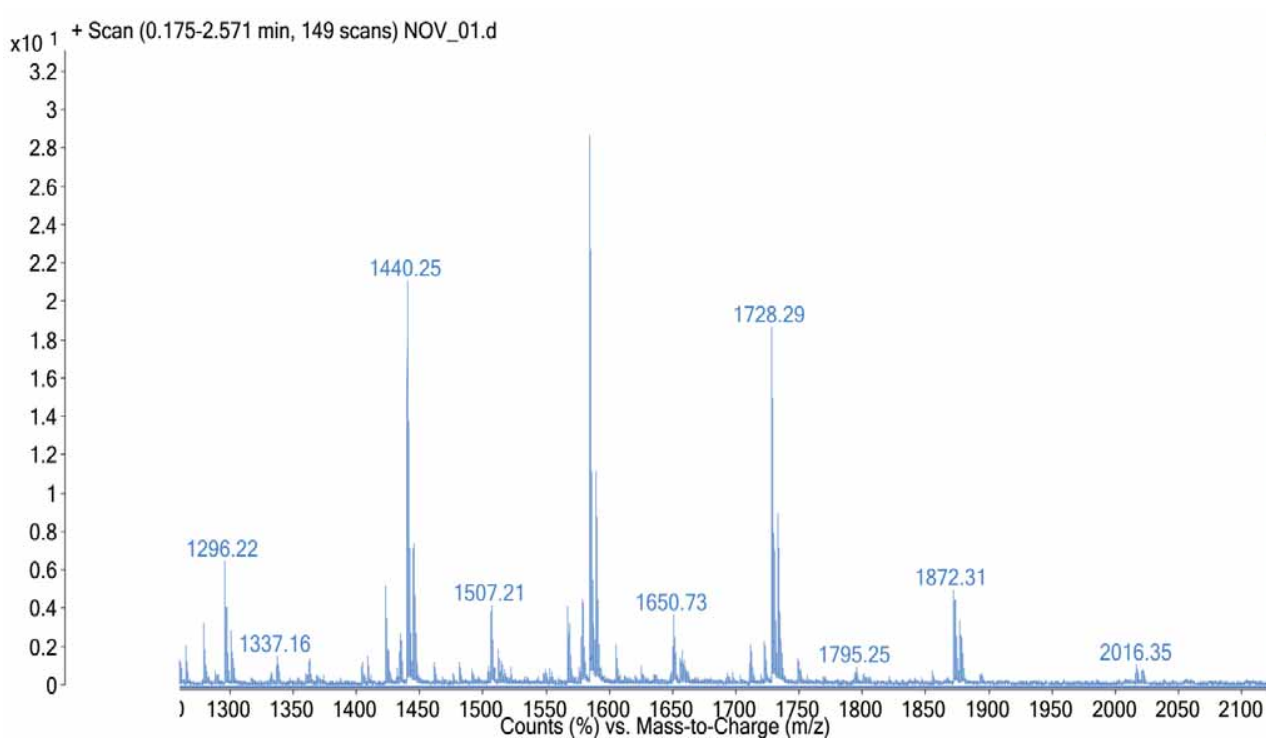

FIGURE 2. ESI MS spectra of the $C D$ - $L A$ derivative

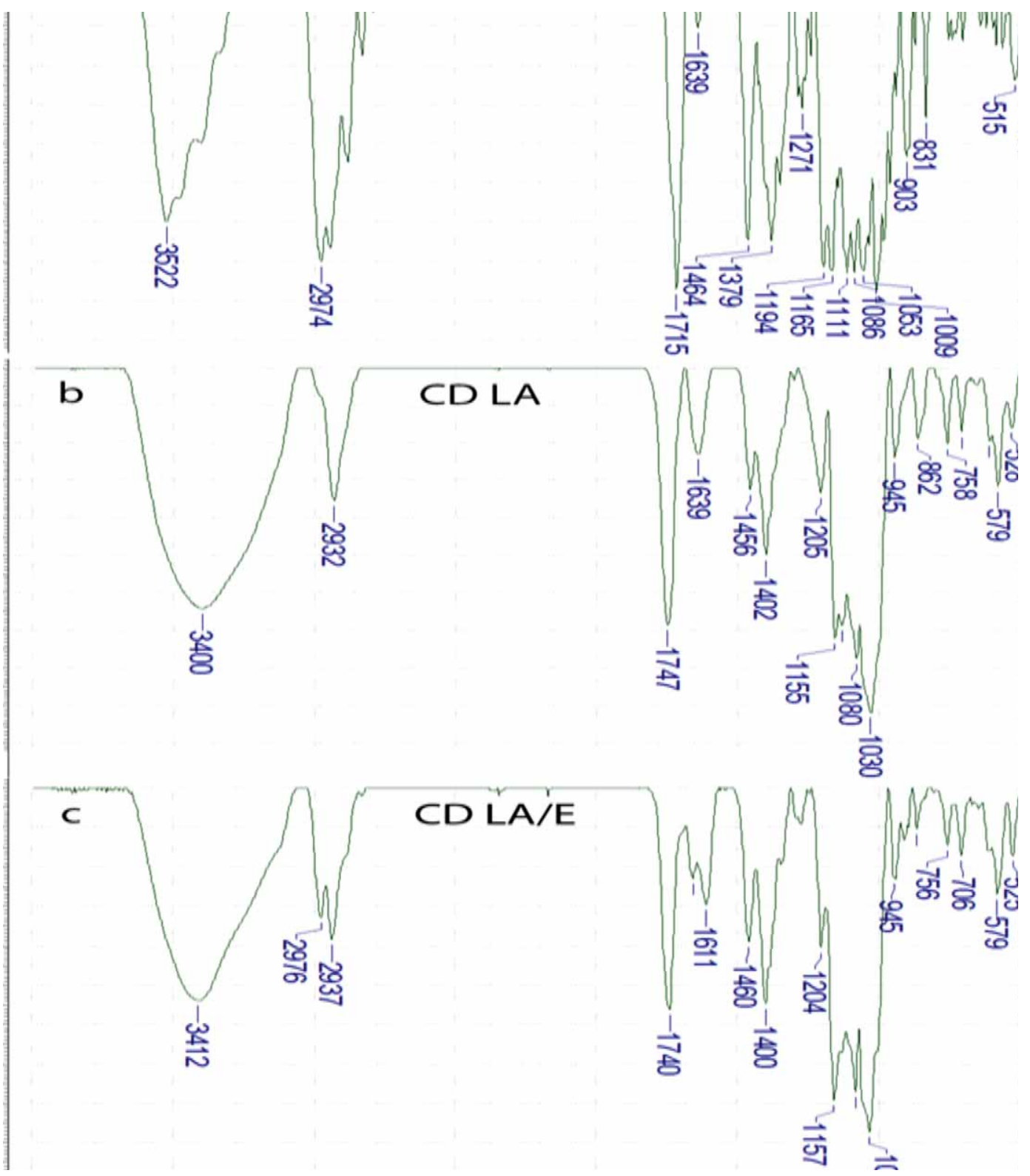

FIGURE 3. FTIR spectrum: ER (a), CD-LA (b), CD-LA_E (c) 


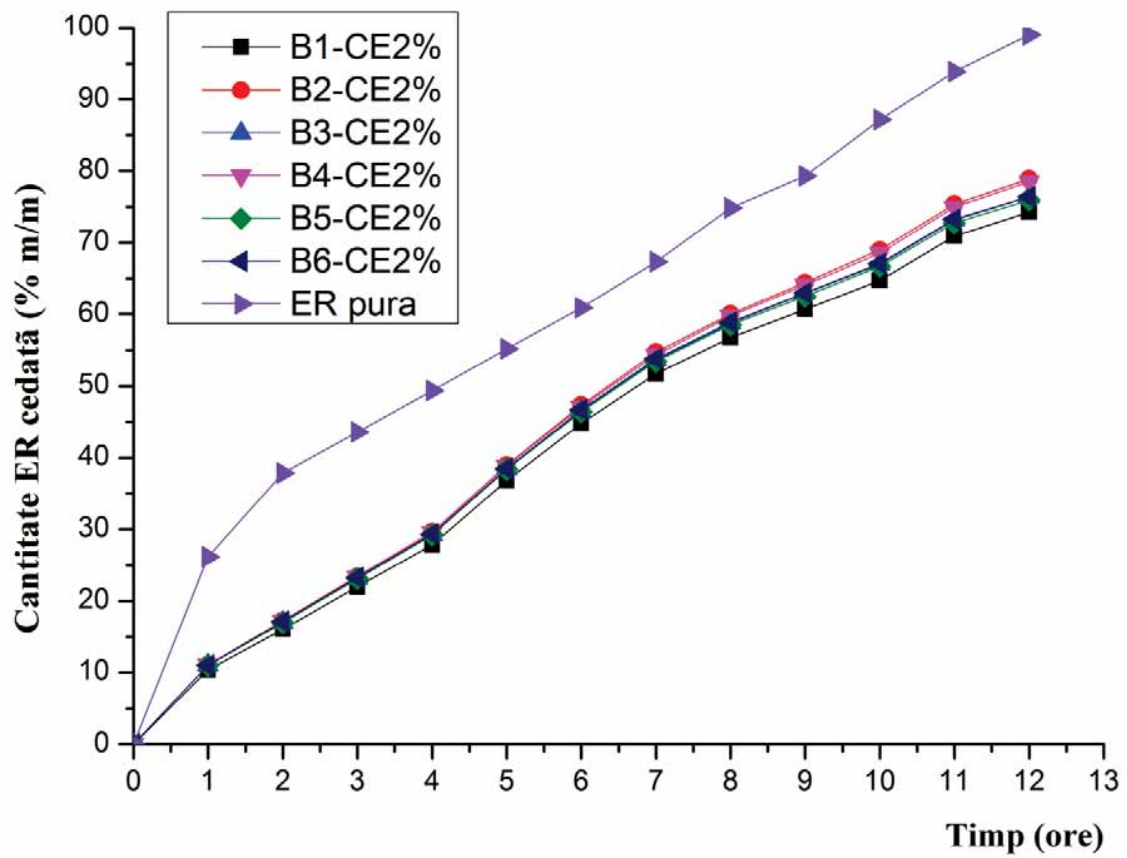

FIGURE 4. Cumulative profile of the in vitro release of ER from gels with CD-LA_E

As shown in Table 2, the permeation coefficient of ER in the CDLA_E complex is greater from the Carbopol-based gel and Lutrol ER 127-based gel. The release kinetics of complexed ER is carried out by diffusion, according to the results obtained by fitting the data from the in vitro dissolution test (table III).

\section{CONCLUSIONS}

The present study synthesized and characterized an ER complex obtained by inclusion in lactide- modified $\beta-C D$. Changing $\beta-C D$ structure aimed at increasing the polarity of the $\mathrm{CD}$ molecule and improving ER binding capacity. Scanning electron spectroscopy showed that by complexation ER alters its crystalline structure and becomes amorphous. The study also showed that specific bands for certain groups in the structure of erythromycin were displaced during the inclusion process. In the structure of the CD-LA_E complex, the molar ratio of CD-LA and ER is 2.12 to 1 . CD-LA_E manifests a good compatibility with a wide range of bases for semi-solid preparations. By complexation, both solubility and permeability of ER were improved. According to the results of the in vitro test, all the formulations studied showed a release of above $75 \%$ over 12 hours. Of the bases studied, the gels based on Carbopol 940 and polyoxyethylene-copolymers, B2-CE2 \% and B4-CE2\%, respectively, exhibited the best characteristics of active principle release.

Conflict of interest: none declared Financial support: none declared

TABLE 2. Parameters specific of ER permeation from CD-LA_E gels $(n=6)$

\begin{tabular}{|c|c|c|}
\hline \multirow{2}{*}{ Formula } & \multicolumn{2}{|c|}{ Permeation parameters } \\
\cline { 2 - 3 } & \multicolumn{2}{|c|}{ Synthetic membrane (in vitro) } \\
\cline { 2 - 3 } & $\boldsymbol{J}_{s s}\left(\boldsymbol{\mu g} / \mathbf{c m}^{2} / \mathbf{h}\right)$ & 7115.86 \\
\hline B1-CE 2\% & $30.4559 \pm 7.2197$ & 7561.58 \\
\hline B2-CE 2\% & $30.3371 \pm 7.5029$ & 5424.43 \\
\hline B3-CE 2\% & $25.0658 \pm 7.7158$ & 7882.27 \\
\hline B4-CE 2\% & $30.3625 \pm 7.7720$ & 6926.99 \\
\hline B5-CE 2\% & $28.2829 \pm 7.7888$ & 6156.37 \\
\hline B6-CE 2\% & $26.9587 \pm 7.4907$ & \\
\hline
\end{tabular}

TABLE 3. Parameters of the analysis of the release kinetics of ER from CD-LA_E gels

\begin{tabular}{|c|c|c|c|c|c|c|c|c|c|}
\hline \multirow[b]{2}{*}{ Formula } & \multicolumn{2}{|l|}{ zero order } & \multicolumn{2}{|l|}{ firstorder } & \multicolumn{2}{|c|}{ Higuchi } & \multicolumn{3}{|c|}{ orsmeyer-Peppas } \\
\hline & $\begin{array}{l}K_{0} \\
g\end{array}$ & $R^{2}$ & $\begin{array}{c}K_{1} \\
\left(h^{-1}\right)\end{array}$ & $R^{2}$ & $\begin{array}{c}K_{H} \\
\left(h^{-0.5}\right)\end{array}$ & $R^{2}$ & $\begin{array}{l}K_{p} \\
h\end{array}$ & $n$ & $R^{2}$ \\
\hline \multicolumn{10}{|c|}{ Synthetic membrane } \\
\hline B1-CE 2\% & 7.5498 & 0.9890 & 0.1357 & 0.9514 & 21.5391 & 0.9434 & 10.4687 & 0.85 & 0.9962 \\
\hline B2-CE 2\% & 7.8527 & 0.9843 & 0.1473 & 0.9495 & 22.4513 & 0.9490 & 10.8949 & 0.85 & 0.9959 \\
\hline B3-CE 2\% & 6.9357 & 0.9759 & 0.1125 & 0.9837 & 19.8826 & 0.9548 & 10.7219 & 0.80 & 0.9947 \\
\hline B4-CE $2 \%$ & 8.0226 & 0.9822 & 0.1547 & 0.9464 & 22.9561 & 0.9515 & 12.3940 & 0.80 & 0.9958 \\
\hline B5-CE $2 \%$ & 7.6682 & 0.9788 & 0.1381 & 0.9656 & 21.9656 & 0.9537 & 11.8511 & 0.80 & 0.9954 \\
\hline B6-CE 2\% & 7.2880 & 0.9783 & 0.1240 & 0.9757 & 20.8812 & 0.9538 & 11.2642 & 0.80 & 0.9954 \\
\hline
\end{tabular}




\section{REFERENCES}

1. Barlet K., Davis S.A., Feldman S.R. et al. Topical antimicrobial acne treatment tolerability: a meaningful factor in treatment adherence? J Am Acad Dermatol 2014; 71: 581-582.

2. Zouboulis C.C., Fischer T.C., Wohlrab J., Barnard J., Alio A.B. Study of the efficacy, tolerability, and safety of 2 fixed-dose combination gels in the management of acne vulgaris. Cutis 2009; 84: 223-229.

3. Del Rosso J.Q., Leyden J.J., Thiboutot D. et al. Antibiotic use in acne vulgaris and rosacea: clinical considerations and resistance issues of significance to dermatologist. Cutis 2008; 82(2S[ii]): 5-12.

4. Giamarellos-Bourboulis E.J. Macrolides beyond the conventional antimicrobials: a class of potent immunomodulators. Int J Antimicrob Agents. 2008; 31: 12-20.

5. Khabriev RU, Popkov VA, Reshetnyak VY, Krasnyuk II. Solubility of erythromicin from solid dispersions. Pharm Chem J. 2009; 43: 36-43.

6. Song W., Yu X., Wang S. Cyclodextrinerythromycin complexes as a drug delivery device for orthopedic application. Int J Nanomedicine 2011; 6: 3173-3186.
7. Shen J., Hao A., Du G. et al. A convenient preparation of 6-oligo(lactic acid) cyclomaltoheptaose as kinetically degradable derivative for controlled release of amoxicillin. Carbohyd Res 2008; 343: 2517-2522

8. Peptu C., Nicolescu A., Peptu C.A. et al. Mass spectrometry characterization of 3-OH butyrated $\beta$-cyclodextrin. J Polym Sci A Polym Chem 2010; 48: 5581-5592.

9. Peptu C., Kwiecień I., Harabagiu V. Modification of $\beta$-ciclodextrin through solution ringopening oligomerization of $\beta$-butirlactone. Cell Chem Technol 2014; 48: 1-10.

10. Miao Y., Zinck P. Ring-opening polymerization of lactones using binaphthyl-diyl hydrogen phosphate as organocatalyst and resulting monosaccharide functionalization of polylactones. Polym Chem 2012; 3: 1119-1122.

11. Socrates G. Infrared and Raman characteristic group frequencies. 3rd ed. New York, John Wiley \& Sons Publishing House. 2004: 109-135.

12. M. Hortolomei, L. Ochiuz, I. Popovici et al. Development and validation of the high performance liquid chromatography method for the quantitative determination of erythromycin in dermo-preparations. Rev. Med. Chir. Soc. Med. Nat., vol. 119, no. 4, pp. $1174-1178$, Dec. 2015

13. A. Azeem, F.J. Ahmad, R.K. Khar, S. Talegaonkar. Nanocarrier for the transdermal delivery of an antiparkinsonian drug. AAPS PharmSciTech, vol. 10, no. 4, pp. 1093 - 1103, Dec. 2009

14. K.T.E. Kierstan, A. Beezer, J.C. Mitchell et al. UV-spectrophotometry study of membrane transport processes with a novel diffusion cell. Int. J. Pharm., vol. 229, no. 1-2, pp. 87-94, Ian. 2001.

15. M.J. Lucero, C. Claro, M. Casas, M.R. Jimenez-Castellanos. Rheological and mechanical properties of hydrophilic dispersions using a new hydrophobically modified polymer and Igepal ${ }^{\circledR} \mathrm{CO} 520$. Carbohyd. Polym., vol. 86, no. 2, pp. 891-896, 2011

16. Peptu C., Harabagiu V. Tandem mass spectroscopy characterization of esterified cyclodextrines. Dig J Nanomater Bios 2013; 8: 1551-1561. 